\title{
Influence of environmental temperature on energy balance, diet-induced thermogenesis and brown fat activity in 'cafeteria'-fed rats
}

\author{
BY NANCY J. ROTHWELL AND MICHAEL J. STOCK* \\ Department of Physiology, St George's Hospital Medical School, Tooting, \\ London SWI7 ORE
}

(Received 9 December 1985 - Accepted 18 March 1986)

1. Young male rats were fed on a pelleted stock diet or a variety of palatable food items ("cafeteria" diet) and housed at $24^{\circ}$ or $29^{\circ}$.

2. 'Cafeteria' feeding at the lower temperature stimulated energy intake, gain and expenditure, but reduced energetic efficiency such that over $70 \%$ of the excess intake was expended.

3. Housing at $29^{\circ}$ suppressed intake and expenditure in animals on both diets, but to a greater extent in 'cafeteria'-fed rats and energetic efficiency was greater than control values at this higher temperature.

4. The thermogenic capacity of brown fat (mitochondrial purine nucleotide binding) was increased by 'cafeteria' feeding, but was suppressed in animals kept at $29^{\circ}$.

5. The results demonstrate that diet-induced thermogenesis is inhibited by high environmental temperatures.

Voluntary food intake and body-weight regulation are highly dependent on environmental temperature and closely related to thermoregulation, particularly in homeotherms (Brobeck, 1948; Hamilton, 1967). Thus Brobeck (1948) was led to the suggestion that food intake was largely determined by the requirement to produce heat for the maintenance of body temperature. It is now obvious that intake control is subject to many diverse influences, but Brobeck's (1948) thermostatic hypothesis does suggest that various relations could exist between energy-balance regulation and thermoregulation. For example, apart from the obligatory changes in metabolic rate following ingestion of food, exposure to cold or hyperphagia also stimulates adaptive increases in heat production known respectively as non-shivering thermogenesis (NST) and diet-induced thermogenesis (DIT). Conversely, it is likely that high environmental temperatures could affect both hyperphagia and the heat produced via DIT, as well as inhibiting NST.

DIT can be readily demonstrated by presenting young rodents with a choice of palatable food items (known as the 'cafeteria' diet) to induce hyperphagia (Rothwell \& Stock, 1979, $1982 a, b$; Trayhurn et al. 1982). 'Cafeteria'-fed rats can show increases in heat production of up to $100 \%$, which apparently result from the same processes as those involved in non-shivering thermogenesis, i.e. sympathetic activation of heat production in brown adipose tissue (BAT) via the mitochondrial proton conductance pathway. These similarities and close relations between NST and DIT are now well described (for reviews see Rothwell \& Stock, 1983; Himms-Hagen, 1985) but, as an example, it has been shown that, compared with normophagic controls, 'cafeteria'-fed rats maintained in a warm environment $\left(24^{\circ}\right)$ show improved cold tolerance when acutely exposed to $5^{\circ}$ (Rothwell \& Stock, 1980). Conversely, cold-adaptation enhances the acute postprandial thermic response to food (Rothwell et al. 1982), and feeding rats on a 'cafeteria' diet in the cold produces larger increases in metabolic rate and BAT activity than seen in stock-fed animals at the same temperature (Rothwell \& Stock, 1980).

* For reprints. 
Most 'cafeteria'-feeding studies have been performed at temperatures of $21-25^{\circ}$, but the findings described previously indicate that while cool environments enhance DIT, warmer temperatures may suppress thermogenesis and promote obesity. We have tested this possibility in the present experiments by studying the effects of 'cafeteria' feeding on energy balance and BAT activity in rats maintained at either $24^{\circ}$ or their thermoneutral temperature, $29^{\circ}$.

\section{EXPERIMENTAL}

Thirty-eight male, Sprague-Dawley rats (Charles River, Kent), aged $35 \mathrm{~d}$, were divided into four groups of eight and one of six with the same mean body-weight, and the latter group (Bo) was killed on day 1 for determination of initial body energy content. The remaining animals were split between two adjacent rooms maintained at either $24 \pm 1^{\circ}$ or $29 \pm 1^{\circ}(12 \mathrm{~h}$ light-12 h dark cycle) and housed for $14 \mathrm{~d}$ with free access to water and a pelleted stock diet (PRD, Christopher Hill Group Ltd, Dorset; metabolizable energy (ME) density $12.0 \mathrm{~kJ} / \mathrm{g}$, protein content $27 \% \mathrm{ME}$ ). Half the rats at each temperature were also fed on a 'cafeteria' diet, comprising four different food items each day. The food items were selected from a list of twenty-five foods, comprising various biscuits, chocolate, cakes, pasta, crisps, meats and pastries. The selection changed every day, but always included a meat or meat product which was fed in the evening at the beginning of the dark cycle (for further details of 'cafeteria'-feeding, see Rothwell \& Stock, 1982a). ME intake was measured as described previously (Rothwell \& Stock, 1982a) from the weight of each food presented (including the stock diet) and its gross energy density minus the energy losses in any spilt food, urine and faeces. Gross energy was determined by ballistic bomb calorimetry of replicate freeze-dried samples.

After $14 \mathrm{~d}$, all rats were killed and the interscapular BAT depot dissected, weighed and homogenized in sucrose before isolation of mitochondria. The binding of $\left[{ }^{3} \mathrm{H}\right]$ guanosine diphosphate $(10 \mathrm{Ci} / \mathrm{mmol}$, Amersham International plc, Amersham, Bucks; GDP) to isolated mitochondria was measured (for details, see Brooks et al. 1982) to assess the activity of the mitochondrial proton conductance pathway (Nicholls \& Locke, 1983). The protein content of whole-tissue homogenates and mitochondrial samples was measured using a coomassie blue dye reagent method (Bio-Rad, Watford).

The carcasses were frozen and gross energy content subsequently determined by ballistic bomb calorimetry (Gallenkamp, Loughborough) of multiple (usually 5-6) homogenized, freeze-dried samples. The coefficient of variation of these determinations ranged from 0.5 to $4.5 \%$. Body energy gain was estimated by subtracting initial body energy content (average of Bo values) from final energy content. Energy expenditure over the experiment was calculated as ME intake minus body energy gain, and gross and net efficiencies expressed as the body energy gain per unit energy, or per unit intake above maintenance (assumed to be $420 \mathrm{~kJ} / \mathrm{kg}$ body-weight $t^{0 \cdot 75}$ per d) respectively.

Values are presented as means with their standard errors, and differences between groups were tested by analysis of variance and Student's $t$ test for unmatched data.

RESULTS

'Cafeteria'-fed rats gained slightly more weight than their respective controls at both temperatures, but these small differences in body-weight and weight gain were not statistically significant (Table 1). ME intake was increased by $32 \%$ in 'cafeteria'-fed rats compared with controls at $24^{\circ}$, and exposure to $29^{\circ}$ suppressed intake in both groups, but to a greater extent in the 'cafeteria'-fed group. Thus the level of hyperphagia was reduced in 'cafeteria'-fed rats at $29^{\circ}(14 \%$ above controls $)$, and was further diminished when values 


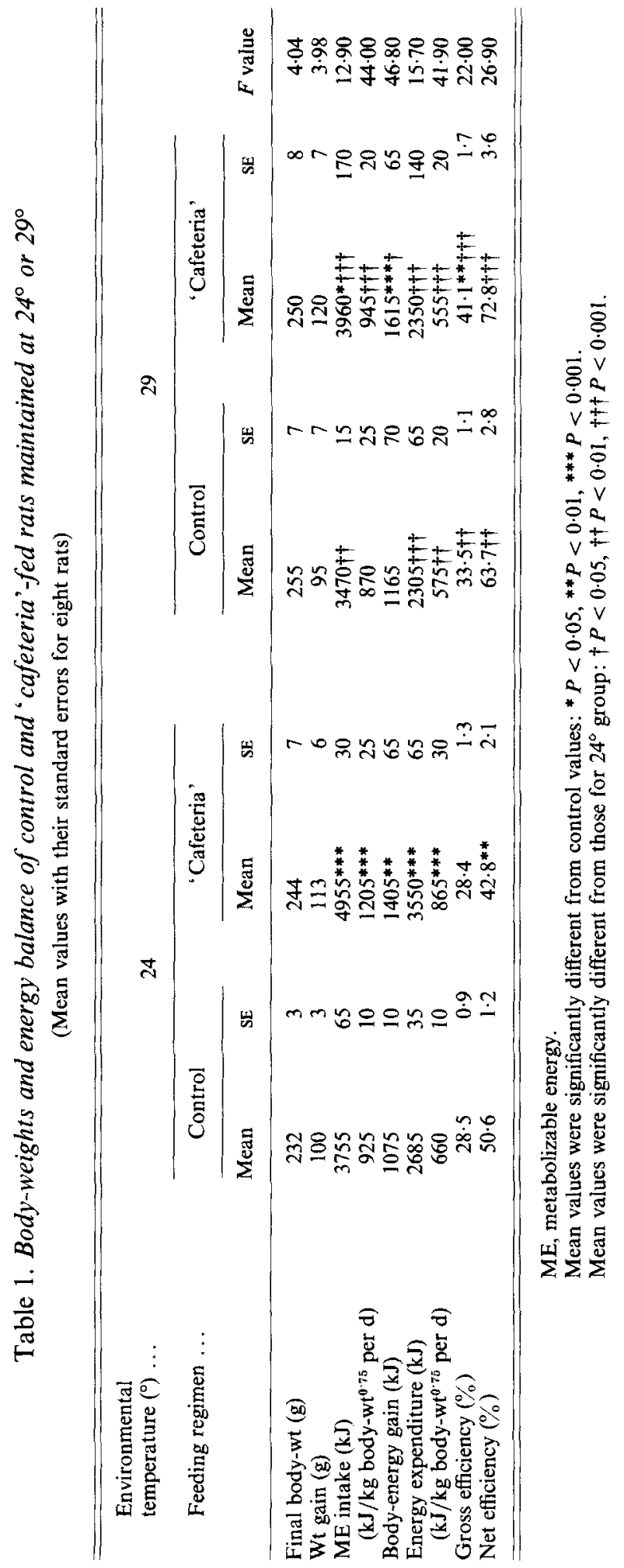




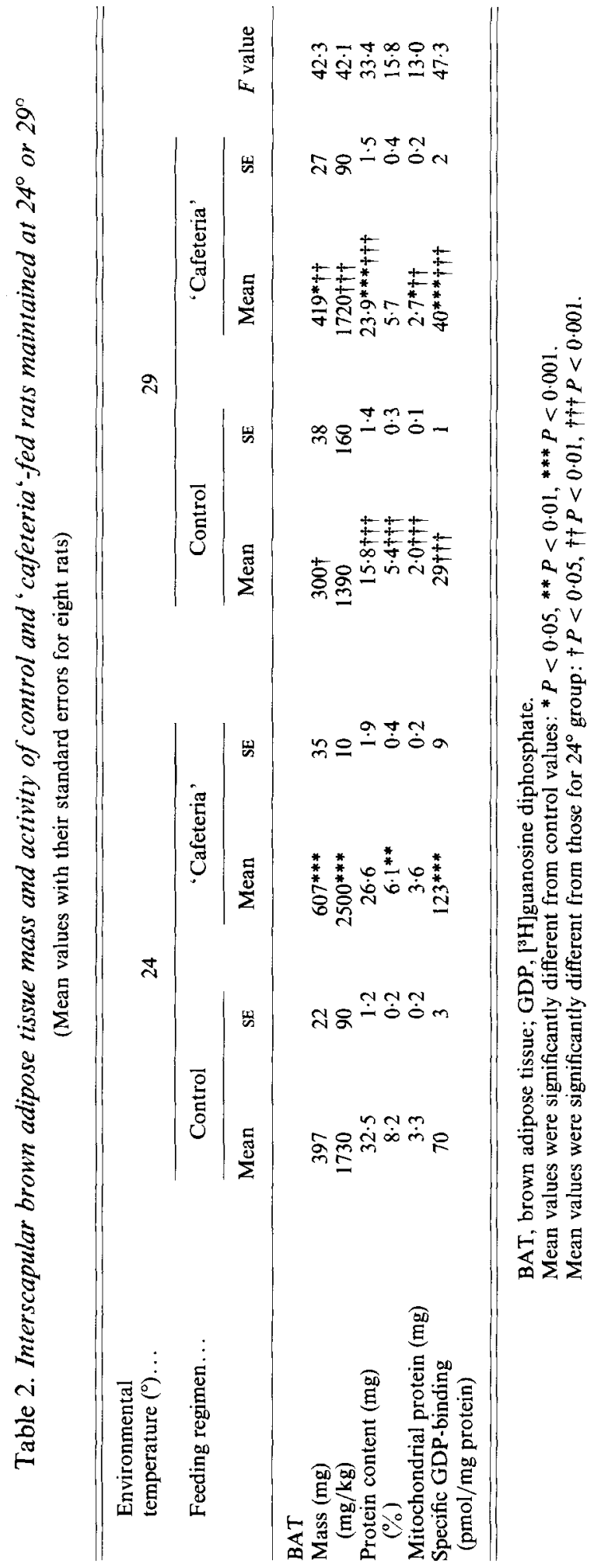


were corrected for body size. Casual observations indicated that food selection and behaviour were unaffected by environmental temperature, although rats housed at $29^{\circ}$ were more often observed to be licking and grooming themselves, possibly indicating some thermal stress.

Body-energy gain (Table 1) was greater in the 'cafeteria'-fed groups, and this was increased further by housing at $29^{\circ}$. Energy expenditure was increased in 'cafeteria'-fed rats kept at the lower temperature $(32 \%$ above control) but was reduced in all animals kept at the high temperature and was unaffected by diet. Gross energetic efficiency was similar for control and 'cafeteria'-fed rats at $24^{\circ}$, but net efficiency was significantly lower in the latter group. At $29^{\circ}$, gross and net energetic efficiencies on both diets were greater than the values obtained at $24^{\circ}$. Thus, unlike the effects seen at $24^{\circ}$, 'cafeteria' feeding at $29^{\circ}$ caused a $23 \%$ increase in gross efficiency and a $14 \%$ (but non-significant) increase in net efficiency.

'Cafeteria' feeding at both temperatures caused hypertrophy of the interscapular BAT depot, i.e. increases in mass and protein content (Table 2), but the absolute values were all very much lower in animals maintained at $29^{\circ}$. The thermogenic activity of BAT, assessed from the binding of GDP to isolated mitochondria, was increased in 'cafeteria'-fed rats at $24^{\circ}\left(76 \%\right.$ above control) and at $29^{\circ}$ (38\% above control), but reduced by over twofold in animals fed on either of the two diets at the higher temperature.

\section{DISCUSSION}

The methods employed in the present study to assess energy balance have been used extensively in previous experiments on 'cafeteria'-fed rats. For example, the three components of energy balance (intake, carcass gain and expenditure) have each been measured by two different methods and shown to agree to within a few per cent (Rothwell \& Stock $1982 a, b)$ in both stock- and 'cafeteria'-fed rats. The results obtained in the present study in rats maintained at $24^{\circ}$ are in general agreement with our own previous work (e.g. Rothwell \& Stock, 1979, 1982a,b; Rothwell et al. 1985) and those of other groups (Stephens et al. 1981; Tulp, 1981; Trayhurn et al. 1982); i.e. 'cafeteria' feeding stimulated energy intake and expenditure and suppressed energetic efficiency. The level of hyperphagia was slightly lower than that usually obtained, possibly because some foods preferred by the rats (e.g. fresh liver) were not used in order to avoid deterioration at the higher temperature.

In spite of the lower level of hyperphagia, over $72 \%(870 \mathrm{~kJ})$ of the excess intake $(1200 \mathrm{~kJ})$ of the 'cafeteria'-fed rats housed at $24^{\circ}$ was expended as heat and less than $30 \%$ was retained in the carcass. This means that the extra energy consumed by the 'cafeteria'-fed rats was utilized with an efficiency of only $28 \%$, which is considerably lower than their overall net energetic efficiency ( $43 \%$ ) and suggests that when extra energy is consumed it is utilized by different metabolic processes. The increased energy expenditure of these animals was accompanied by a $76 \%$ increase in BAT mitochondrial GDP-binding capacity, and in earlier experiments we have confirmed that these changes are associated with increases in BAT noradrenaline turnover (Young et al. 1982) and greater in vivo oxygen consumption of the tissue (Rothwell \& Stock, 1981).

At $29^{\circ}$, rats fed on both diets showed lower levels of energy intake and expenditure, but due to an overall increase in energetic efficiency the rates of body-energy gain were slightly (control) or significantly ('cafeteria') greater than in those housed at $24^{\circ}$. In addition to the general effects of the higher ambient temperature on the level of metabolism, exposure to $29^{\circ}$ also affected the magnitude of the response to 'cafeteria' feeding by attenuating the increases in intake and expenditure. Also, the higher temperature produced an increase, rather than a decrease in energetic efficiency in 'cafeteria'-fed rats. Unlike the 'cafeteria'-fed rats housed at $24^{\circ}$, only $8 \%(40 \mathrm{~kJ})$ of the excess intake $(490 \mathrm{~kJ})$ was dissipated as heat in 
those maintained at the higher temperature. Thus the net efficiency of utilization of this extra energy was $92 \%$ (cf. $28 \%$ at $24^{\circ}$ ). As might be expected, housing the animals at thermoneutrality markedly suppressed the activity of the proton conductance pathway (i.e. GDP-binding) in BAT mitochondria and, although 'cafeteria' feeding produced an increase, the absolute level was considerably lower than that seen in control and 'cafeteria'-fed animals at $24^{\circ}$.

Theoretically, the efficiency of energy utilization for body-energy (i.e. fat) gain should increase when animals are fed on the 'cafeteria' diet, which is typically high-fat (over $40 \%$ of ME) compared with the stock diet (less than 10\% of ME). However, it is apparent from the present and previous studies conducted at $24^{\circ}$ that the energy spared on the cost of lipogenesis as a result of the greater intake of dietary lipid is more than offset by adaptive increases in heat production. This results in either no change or even a decrease in energetic efficiency. Inhibition of DIT (to avoid hyperthermia, for example) would prevent any adaptive decrease in energetic efficiency, thus unmasking the predictable effects of feeding high-fat diets on the cost of carcass energy gain and producing an increase in energetic efficiency. It could be argued that the failure to observe adaptive DIT in 'cafeteria'-fed rats at $29^{\circ}$ was simply due to the absence of hyperphagia. However, it should be noted that, whereas energy expenditure was practically identical in control and 'cafeteria'-fed rats at $29^{\circ}$, voluntary food intake was still elevated in the 'cafeteria'-fed group. This suggests that heat production was strictly determined by thermoregulatory requirements, whereas the hedonistic properties of the 'cafeteria' diet prevailed, to produce the most damaging combination for the regulation of energy balance: hyperphagia plus hyper-efficiency.

We have previously reported a close relation between low environmental temperatures and DIT in'cafeteria'-fed rats, and demonstrated that 'cafeteria'-fed rats show improved cold tolerance and a greater capacity for DIT during chronic exposure to the cold (Rothwell \& Stock, 1980). These interactions between the two stimuli are to be expected in view of the common mechanisms shared by NST and DIT, but they may operate at several levels. For example, environmental temperature may influence thermogenesis via effects on peripheral thermoreceptors and the hypothalamic pre-optic thermoregulatory centres, with secondary changes in energy intake compensating for alterations in the level of expenditure. Alternatively, some of the increased heat production during cold exposure could be secondary to cold-induced increases in food intake, thus producing DIT. The involvement of the ventromedial hypothalamus (VMH) in both the control of food intake and BAT thermogenesis (see Rothwell \& Stock, 1983) and the influence of pre-optic cooling on VMH activity and BAT thermogenesis (Imai-Matsumura et al. 1984) suggests that central mechanisms exist to allow a complex interaction between dietary and thermal stimuli.

The present results have not only demonstrated that DIT in 'cafeteria'-fed rats can be inhibited at high environmental temperatures, but may also explain some apparent anomalies between our own studies and those of other workers. Barr \& McCracken (1984), for example, reported a high efficiency of energy utilization in 'cafeteria'-fed rats kept at $29^{\circ}$ and suggested that this conflicted with our previous observations of increased DIT and reduced efficiency during 'cafeteria' feeding. It is now obvious that conducting experiments at high environmental temperatures is likely to suppress DIT and should not be used to assess its quantitative importance in animals housed at lower ambient temperatures. It would also seem that the thermoneutral zone and the lower critical temperature of hyperphagic rats exhibiting DIT will be considerably lower than in normophagic stock-fed rats. This effect of the plane of nutrition on critical temperature has been described previously in the pig (Close \& Mount, 1978). 
The authors are grateful to Mike Lacey, Ian Connoley and Kevin Bryant for their technical assistance. This work was supported by grants from ICI plc and a Royal Society University Fellowship to N.J.R.

\section{REFERENCES}

Barr,H. G. \& McCracken, K. J. (1984). British Journal of Nutrition 51, 379-387.

Brobeck, J. R. (1948). Yale Journal of Biological Medicine 20, 545-552.

Brooks, S. L., Rothwell, N. J. \& Stock, M. J. (1982). Quarterly Journal of Experimental Physiology 67, $259-268$.

Close, W. H. \& Mount, L. E. (1978). British Journal of Nutrition 40, 413-421.

Hamilton, C. L. (1967). In Handbook of Physiology, Section 6, Alimentary Canal, vol. 1. Food and Water Intake, pp. 303-317 [C. F. Code, editor]. Washington DC: American Physiological Society.

Himms-Hagen, J. (1985). Annual Review of Nutrition 5, 69-94.

Imai-Matsumura, K., Matsumura, K. \& Nakayama, T. (1984). Japanese Journal of Physiology 34, 939-943.

Nicholls, D. G. \& Locke, R. (1983). Physiological Reviews 64, 1-64.

Rothwell, N. J., Saville, M. E. \& Stock, M. J. (1982). International Journal of Obesity 6, 53-59.

Rothwell, N. J. \& Stock, M. J. (1979). Nature 281, 31-35.

Rothwell, N. J. \& Stock, M. J. (1980). Canadian Journal of Physiology and Pharmacology 58, 842-848.

Rothwell, N. J. \& Stock, M. J. (1981). Pfiügers Archiv 389, 237-242.

Rothwell, N. J. \& Stock, M. J. (1982a). British Journal of Nutrition 47, 461-471.

Rothwell, N. J. \& Stock, M. J. (1982 b). Journal of Physiology 328, 371-377.

Rothwell, N. J. \& Stock, M. J. (1983). In Mammalian Thermogenesis, pp. 208-233 [L. Girardier and M. J. Stock, editors]. London: Chapman \& Hall.

Rothwell, N. J. \& Stock, M. J. (1984). In Recent Advances in Physiology, vol. 10, pp. 349-384 [P. F. Baker, editor]. Edinburgh: Churchill Livingstone.

Rothwell, N. J., Stock, M. J. \& Warwick, B. P. (1985). Metabolism 34, 474-480.

Stephens, D. N., Nash, S. C. \& Proffitt, C. (1981). Pflügers Archiv 392, 7-12.

Trayhurn, P., Jones, P. M., McGuckin, M. M. \& Goodbody, A. E. (1982). Nature 295, 323-325.

Tulp, O. L. (1981). International Journal of Obesity 5, 579-591.

Young, J. B., Saville, M. E., Rothwell, N. J., Stock, M. J. \& Landsberg, L. (1982). Journal of Clinical Investigation 69, 1061-1071: 